https://helda.helsinki.fi

\title{
Scaling Density of Axion Strings
}

\section{Hindmarsh, Mark}

2020-01-16

Hindmarsh , M , Lizarraga , J , Lopez-Eiguren , A \& Urrestilla , J 2020 , ' Scaling Density of Axion Strings ' , Physical Review Letters , vol. 124 , no. 2 , 021301 . https://doi.org/10.1103/PhysRevLett.124.02130

http://hdl.handle.net/10138/313457

https://doi.org/10.1103/PhysRevLett.124.021301

unspecified

publishedVersion

Downloaded from Helda, University of Helsinki institutional repository.

This is an electronic reprint of the original article.

This reprint may differ from the original in pagination and typographic detail.

Please cite the original version. 


\title{
Scaling Density of Axion Strings
}

\author{
Mark Hindmarsh $\odot,{ }^{1,2, *}$ Joanes Lizarraga $\oplus^{3, \dagger}$ Asier Lopez-Eiguren $\oplus^{1, \$}$ and Jon Urrestilla $\oplus^{3, \S}$ \\ ${ }^{1}$ Department of Physics and Helsinki Institute of Physics, University of Helsinki, PL 64, FI-00014, Finland \\ ${ }^{2}$ Department of Physics and Astronomy, University of Sussex, Falmer, Brighton BN1 9QH, United Kingdom \\ ${ }^{3}$ Department of Theoretical Physics, University of the Basque Country UPV/EHU, 48080 Bilbao, Spain
}

(Received 7 October 2019; published 16 January 2020)

\begin{abstract}
In the QCD axion dark matter scenario with postinflationary Peccei-Quinn symmetry breaking, the number density of axions, and hence the dark matter density, depends on the length of string per unit volume at cosmic time $t$, by convention written $\zeta / t^{2}$. The expectation has been that the dimensionless parameter $\zeta$ tends to a constant $\zeta_{0}$, a feature of a string network known as scaling. It has recently been claimed that in larger numerical simulations $\zeta$ shows a logarithmic increase with time, while theoretical modeling suggests an inverse logarithmic correction. Either case would result in a large enhancement of the string density at the QCD transition, and a substantial revision to the axion mass required for the axion to constitute all of the dark matter. With a set of new simulations of global strings, we compare the standard scaling (constant- $\zeta$ ) model to the logarithmic growth and inverse-logarithmic correction models. In the standard scaling model, by fitting to linear growth in the mean string separation $\xi=t / \sqrt{\zeta}$, we find $\zeta_{0}=1.19 \pm 0.20$. We conclude that the apparent corrections to $\zeta$ are artifacts of the initial conditions, rather than a property of the scaling network. The residuals from the constant- $\zeta$ (linear $\xi$ ) fit also show no evidence for logarithmic growth, restoring confidence that numerical simulations can be simply extrapolated from the Peccei-Quinn symmetry-breaking scale to the QCD scale. Reanalysis of previous work on the axion number density suggests that recent estimates of the axion dark matter mass in the postinflationary symmetry-breaking scenario we study should be increased by about $50 \%$.
\end{abstract}

DOI: 10.1103/PhysRevLett.124.021301

Introduction.-The Peccei-Quinn (PQ) mechanism, which solves the strong $C P$ problem of $\mathrm{QCD}$ by extending the standard model with an extra U(1) global symmetry [1], brings with it a long-lived pseudoscalar particle, the axion [2]. A universe where light axions $[3,4]$ constitute the dark matter [5] is one of the most promising scenarios in the current cosmological paradigm.

If the PQ symmetry is spontaneously broken after primordial inflation, axion strings are formed [6], a variety of global cosmic string $[7,8]$. They survive until the QCD confinement transition, when they become connected by domain walls made of the $C P$-odd gluon condensate $[9,10]$ and are annihilated. Most of the energy is left behind in the form of axion radiation, produced through the lifetime of the string network and during the annihilation phase. The axion radiation can also be viewed as light massive particles, whose number density depends on the length of string per unit volume $\zeta / t^{2}$, where $t$ is cosmic time. The important dimensionless parameter $\zeta$ can be established only by numerical simulations.

The usual expectation (see Refs. [11-13]) is that the string density parameter $\zeta$ converges to a constant within a few Hubble times after the network is formed, part of a wider assumption known as scaling. Scaling means that the string network is statistically self-similar; i.e., all macroscopic quantities with the dimensions of length and time are proportional to the Hubble length and time. Earlier simulations of global cosmic strings [14-21] were consistent with scaling with $\zeta \sim 1$, and there is good theoretical understanding of scaling from modeling the global properties of the network [22,23].

However, several groups have recently claimed that $\zeta$ shows a logarithmic increase with time [24-27]. An argument for expecting a scaling violation is based on the logarithmic growth in the effective string tension of a global string with their mean separation. If there is no corresponding change in the energy loss rate per unit length, the string length density parameter should grow [28-31].

In fact, this argument does not lead to logarithmic growth of $\zeta$; instead it gives a leading correction to scaling of an inverse logarithm [32]. Nonetheless, either behavior would lead to a larger asymptotic string density parameter, which would lead to an increase of the axion number density, and hence a decrease in the axion mass required to match the current dark matter mass density.

In this work we present results from a new set of numerical simulations of global strings. We explore the effect of different initial string densities and lattice sizes. We compare the results for the string density in three different two-parameter models defined below: standard scaling, logarithmic, and inverse logarithmic. We demonstrate that all simulations are consistent with standard 
scaling and determine the asymptotic string length density parameter $\zeta_{0}$ to the best precision to date.

We conclude that the axion string density shows excellent scaling following the PQ phase transition, justifying a constant- $\zeta$ extrapolation to the QCD transition. We reexamine previous results to see how estimates of the axion number density, and hence the axion dark matter mass, are affected.

Model and simulations. - The simplest axion models $[3,4]$ break the $\mathrm{U}(1)_{\mathrm{PQ}}$ symmetry with a scalar gauge singlet field, which we can write as a real scalar doublet $\Phi$ with action

$$
S=\int d^{4} x \sqrt{-g}\left(\frac{1}{2} \partial_{\mu} \Phi \partial^{\mu} \Phi-\frac{1}{4} \lambda\left(\Phi^{2}-\eta^{2}\right)^{2}\right),
$$

where $\lambda$ is the self-coupling of the scalar field and $\eta$ its vacuum expectation value. The metric $g_{\mu \nu}$ is the spatially flat Friedmann-Lemaître-Robertson-Walker metric with comoving spatial coordinates $d s^{2}=d t^{2}-a^{2}(t) d \boldsymbol{r}^{2}$, where $a(t)$ is the scale factor and $t$ is physical time.

When PQ symmetry is spontaneously broken, axion strings are formed and one massless Goldstone boson and one massive boson arise. Even though the axions acquire a small mass, when the coupling to QCD fields are considered [1], at high temperatures the axion mass can be neglected and the field obeys the following dynamics:

$$
\Phi^{\prime \prime}+2 \frac{a^{\prime}}{a} \Phi^{\prime}-\nabla^{2} \Phi=-a^{2} \lambda\left(\Phi^{2}-\eta^{2}\right) \Phi,
$$

where the primes represent derivatives with respect to the conformal time $\tau=\int d t a^{-1}(t)$. For axion string evolution, $a \propto \tau$.

The evolution of the field is simulated with a discretized version of Eq. (2), parallelized using the LATfield2 library [33]. We use cubic lattices with periodic boundary conditions, which impose an upper limit in the dynamical range of the simulation of half a light-crossing time, beyond which it is possible for the Goldstone modes to show finite volume effects in their propagation. Note that we do not use the Press-Ryden-Spergel method [34]; data are taken while the string core has constant physical width $r_{s}=m_{s}^{-1}$ and shrinking comoving width.

We use initial conditions designed to drive the system quickly to scaling. To this end, a satisfactory initial field configuration is given by the scalar field velocities $\dot{\Phi}$ set to zero and the components of $\Phi$ to be Gaussian random fields with power spectrum, $P_{\Phi}(\mathbf{k})=A\left[1+\left(k \ell_{\phi}\right)^{2}\right]^{-1}$, with $A$ chosen so that $\left\langle\Phi^{2}\right\rangle=\eta^{2}$. We use comoving correlation lengths $\ell_{\phi} \eta=(5,10,20)$. We run with lattice sites per side $N=[1 k, 2 k, 4 k]$ (where $k=1024)$ and perform four independent runs in each different lattice and for each correlation length.
In order to remove energy from the initial configuration, $\lambda$ is time dependent in the preparation phase, so that we can arrange $m_{s} a \simeq 2 \eta$ at $\tau_{\text {ini }} \eta=50$ and apply a period of diffusive evolution with unit diffusion constant, until $\tau_{\text {diff }} \eta=70$. We then apply the second order equations (2), allowing the comoving width of the strings to grow to their physical value at $\tau_{\mathrm{cg}} \eta=[144.9,196.2,271.1]$ for $N=[1 k, 2 k, 4 k]$.

The physical evolution begins at $\tau_{\mathrm{cg}}$ and ends at $\tau_{\text {end }} \eta=[300,550,1050]$, when $m_{s} a=2 \eta$, during which $\lambda=2$ is constant. We normalize the scale factor so that $a\left(\tau_{\text {end }}\right)=1$. The comoving lattice spacing is $\delta x \eta=0.5$, the conformal time step during diffusion is $\delta \tau=\delta x / 30$ and during second order evolution it is $\delta \tau=\delta x / 5$. In all figures and tables the unit of length is $\eta^{-1}$.

Measurements and results.-The evolution of the string network can be tracked by the mean string separation $\xi$, defined in terms of the mean string length $\ell_{s}$ in the simulation volume $\mathcal{V}$ as

$$
\xi=\sqrt{\mathcal{V} / \ell_{s}} .
$$

The physical string length $\ell_{s}$ is the number of plaquettes pierced by strings multiplied by the physical lattice spacing $a \delta x$, corrected by a factor of $2 / 3$ to compensate for the Manhattan effect [28]. Such plaquettes are identified calculating the "winding" of the phase of the field around each plaquette of the lattice [35].

A dimensionless measure of the length of string per unit volume $[8,24-26,30,32]$ is

$$
\zeta=\ell_{s} t^{2} / \mathcal{V}=t^{2} / \xi^{2},
$$

which in a radiation-dominated universe is 4 times the number of Hubble lengths of string per Hubble volume (note that some authors use $\xi$ to denote this quantity).

As there is no fixed length scale in the string equations of motion, string networks are expected to evolve toward a self-similar or scaling regime, in which the only length scale is $t[7,8,22]$. Hence, $\xi$ should increase linearly with time and $\zeta$ should evolve toward a constant. However, the formation and initial evolution of the network introduces a timescale, which can be taken to be the $t$-axis intercept of a linear fit to $\xi(t)$ [36]. We call this the initial string evolution parameter and denote it $t_{0}$. Over cosmological timescales the ratio $t_{0} / t \rightarrow 0$; however, in numerical simulations it must be taken into account when extracting the scaling value of $\zeta$, which we denote $\zeta_{0}$.

Figure 1 shows the results for the mean string separation $\xi$ for $4 k$ simulations with different initial correlation lengths. Graphs of $\xi$ against $t$ for all runs are shown in the Supplemental Material [37]. Consistent with our earlier simulations [21], after a relatively short period of relaxation, $\xi$ asymptotes to a line that can be well fitted with (note that $\beta$ as defined here is the slope of the 


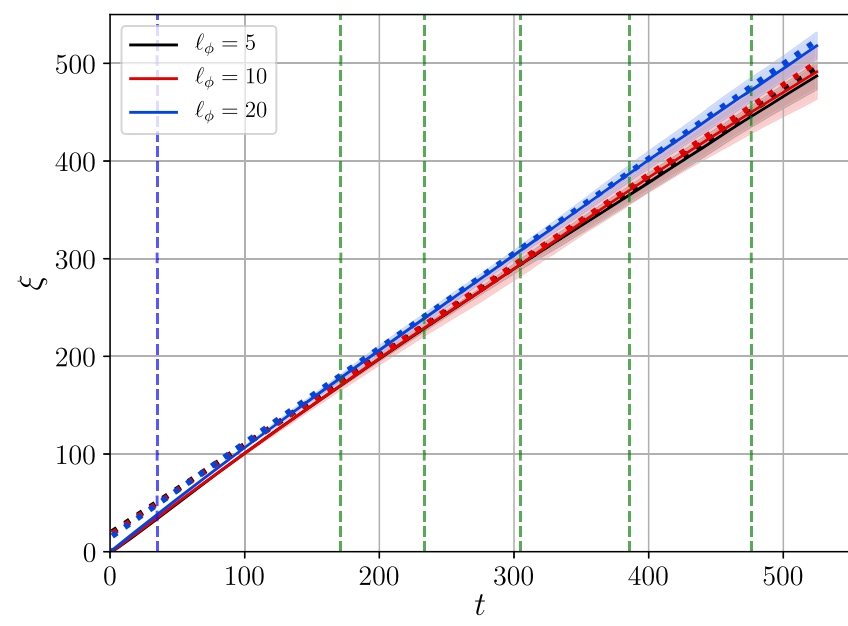

FIG. 1. Mean string separation $\xi$ [defined in Eq. (3)] from $4 k$ simulations with all initial field correlation lengths $\ell_{\phi}$. The solid line represents the mean over realizations of $\xi$ at each time, with the shaded regions showing the $1 \sigma$ variation. Also shown as dotted lines are the linear fits to the form of Eq. (5), whose parameters and uncertainties are shown in Table I. The blue vertical dashed line is the end of the core growth period $\left(t_{\mathrm{cg}}\right)$, after which strings maintain their physical width, and the green ones are the boundaries of the fitting ranges.

comoving string separation $\xi / a$ plotted against conformal time $\tau=2 t / a$ )

$$
\xi=2 \beta\left(t-t_{0}\right) .
$$

This is the standard scaling model. The scaling value of the length density parameter is $\zeta_{0}=1 / 4 \beta^{2}$.

We measure the parameters $\beta$ and $t_{0}$ with a linear fit over four ranges in conformal time, defined by a vector of boundary times $\tau_{b}=(6,7,8,9,10) \tau_{s}$ and $\tau_{s} \eta=[25,50$, $100]$ for $N=[1 k, 2 k, 4 k]$. We choose times in the last half of the conformal time range to minimize biases from the initial conditions. The standard deviation of the central values of the parameters in the different fit ranges can be used to give an estimate of the combined uncertainty due to the approach to scaling and the lattice spacing: later fits will be closer to the scaling value, but more affected by the lattice spacing, which is equal to the inverse mass $(2 \eta)^{-1}$ at the end of the simulation. The standard deviation of the central values between different $\ell_{\phi}$ gives an estimate of the uncertainty due to the initial correlation length. The two uncertainties are added in quadrature to give an estimate of the systematic error $\Delta \beta_{\text {syst }}$, which is dominated by the uncertainty due to the variation in initial correlation lengths. The total uncertainty is obtained from adding the statistical and systematic uncertainties in quadrature. The means and uncertainties for the standard scaling parameters can be found in Tables I and II.

We now turn to the alternative models recently put forward: logarithmic [24-27] and inverse-logarithmic [32] correction to scaling,
TABLE I. Numerical values of the fit parameters for the $4 k$ runs fitted over the conformal time ranges given after Eq. (5), shown in physical time as $\Delta t_{\text {fit }}$. The fit parameters $t_{0}$ and $\beta$ pertain to Eq. (5), with $\zeta_{0}=1 / 4 \beta^{2}$. The values are computed averaging over the 4 different realizations, and the computation of the uncertainties is described after Eq. (5).

\begin{tabular}{lrccc}
\hline \hline$\Delta t_{\text {fit }}$ & $\ell_{\phi}$ & $t_{0}$ & $\beta$ & $\zeta_{0}$ \\
\hline $171.42-233.33$ & 5 & $-8.94 \pm 2.74$ & $0.47 \pm 0.01$ & $1.12 \pm 0.05$ \\
$171.42-233.33$ & 10 & $-7.48 \pm 8.47$ & $0.48 \pm 0.03$ & $1.11 \pm 0.15$ \\
$171.42-233.33$ & 20 & $-10.00 \pm 1.81$ & $0.49 \pm 0.01$ & $1.04 \pm 0.05$ \\
$233.33-304.76$ & 5 & $-16.46 \pm 10.27$ & $0.46 \pm 0.02$ & $1.20 \pm 0.11$ \\
$233.33-304.76$ & 10 & $-19.64 \pm 4.54$ & $0.45 \pm 0.02$ & $1.22 \pm 0.13$ \\
$233.33-304.76$ & 20 & $-12.59 \pm 13.29$ & $0.49 \pm 0.02$ & $1.06 \pm 0.09$ \\
$304.76-385.71$ & 5 & $-29.83 \pm 11.13$ & $0.44 \pm 0.02$ & $1.30 \pm 0.13$ \\
$304.76-385.71$ & 10 & $-12.32 \pm 20.42$ & $0.47 \pm 0.03$ & $1.16 \pm 0.16$ \\
$304.76-385.71$ & 20 & $-12.93 \pm 15.52$ & $0.49 \pm 0.03$ & $1.07 \pm 0.12$ \\
$385.71-476.19$ & 5 & $-27.32 \pm 27.07$ & $0.44 \pm 0.03$ & $1.28 \pm 0.17$ \\
$385.71-476.19$ & 10 & $-34.48 \pm 39.63$ & $0.45 \pm 0.05$ & $1.31 \pm 0.31$ \\
$385.71-476.19$ & 20 & $-23.79 \pm 16.37$ & $0.47 \pm 0.03$ & $1.12 \pm 0.13$ \\
\hline \hline
\end{tabular}

$\zeta(t)=\zeta_{0}^{*}+\alpha^{*} \log (\eta t), \quad \zeta(t)=\zeta_{0}^{\prime}+\alpha^{\prime} / \log (\eta t)$,

where $\zeta_{0}^{*}, \zeta_{0}^{\prime}, \alpha^{*}$, and $\alpha^{\prime}$ are the fitting parameters. We performed fits over the four ranges used previously, using the same method to estimate uncertainties. The mean values and uncertainties for the parameters can be found in Table III.

The uncertainties include zero, and are apparently inconsistent with reports of a logarithmic correction with coefficient $\alpha^{*} \simeq 0.2[24,25]$. It is interesting to examine why. If the strings are scaling in the sense that the mean string separation $\xi$ is increasing linearly, the string density parameter $\zeta$ behaves as

$$
\zeta=\frac{t^{2}}{4 \beta^{2}\left(t-t_{0}\right)^{2}} \simeq \zeta_{0}\left(1+2 \frac{t_{0}}{t}\right) .
$$

The uncorrected estimator approaches its asymptotic value slowly, resembling the behavior of a logarithm with a positive coefficient,

$$
\alpha^{*}\left(t_{f}\right)=-2 \zeta\left(t_{0} / t_{f}\right)\left(1-t_{0} / t_{f}\right)^{-1}
$$

TABLE II. Central values and estimated uncertainties of the standard scaling parameters $\beta$ and $\zeta_{0}=1 / 4 \beta^{2}$ for all box sizes. The decomposition into statistical and systematic uncertainties, as discussed in the text after Eq. (5), is also shown.

\begin{tabular}{ccccccc}
\hline \hline$N$ & $\beta \pm \Delta \beta$ & $\Delta \beta_{\text {stat }}$ & $\Delta \beta_{\text {syst }}$ & $\zeta_{0} \pm \Delta \zeta_{0}$ & $\Delta \zeta_{0, \text { stat }}$ & $\Delta \zeta_{0, \text { syst }}$ \\
\hline $1 k$ & $0.499 \pm 0.042$ & 0.031 & 0.028 & $1.02 \pm 0.17$ & 0.13 & 0.11 \\
$2 k$ & $0.486 \pm 0.036$ & 0.030 & 0.019 & $1.07 \pm 0.16$ & 0.13 & 0.08 \\
$4 k$ & $0.467 \pm 0.037$ & 0.030 & 0.021 & $1.17 \pm 0.20$ & 0.17 & 0.11 \\
\hline \hline
\end{tabular}


TABLE III. Numerical values of the fit parameters of the logarithmic correction, inverse-logarithmic correction, and residuals as presented in Eqs. (6) and (10), respectively. Fitting ranges and error estimates were obtained following the same prescription as in the previous tables.

\begin{tabular}{ccccccc}
\hline \hline$N$ & $\zeta_{0}^{*}$ & $\alpha^{*}$ & $\zeta_{0}^{\prime}$ & $\alpha^{\prime}$ & $\zeta_{r}\left(\times 10^{-2}\right)$ & $\alpha_{r}\left(\times 10^{-2}\right)$ \\
\hline $1 k$ & $1.7 \pm 1.0$ & $-0.14 \pm 0.21$ & $0.55 \pm 0.69$ & $2.4 \pm 3.3$ & $0.0 \pm 1.3$ & $-0.02 \pm 0.31$ \\
$2 k$ & $0.88 \pm 0.60$ & $0.03 \pm 0.11$ & $1.18 \pm 0.58$ & $-0.8 \pm 3.0$ & $0.2 \pm 1.6$ & $-0.04 \pm 0.33$ \\
$4 k$ & $0.42 \pm 0.59$ & $0.11 \pm 0.11$ & $1.66 \pm 0.68$ & $-3.6 \pm 3.8$ & $0.2 \pm 1.5$ & $-0.03 \pm 0.26$ \\
\hline \hline
\end{tabular}

where $t_{f}$ is a time at which the fit is carried out. We find that taking $t_{f}$ to be the final time in the fit range gives the best fit. If $t_{0}<0$, the approach is from lower ("underdense") values of $\zeta$, giving positive values of $\alpha^{*}$, and vice versa. Hence an apparent logarithmic growth parameter $\alpha^{*} \simeq 0.2$ $[24,25]$ is produced for runs where the initial string configurations are biased toward $t_{0} / t_{f} \simeq-0.1$. Our initial conditions cover both positive and negative values of $\alpha^{*}$ and are consistent with $\alpha^{*}=0$ as $t_{0} / t_{f} \rightarrow 0$. The parameter $\alpha^{\prime}$ similarly takes both signs and is consistent with zero as $t_{0} / t_{f} \rightarrow 0$. The constant terms in the alternative models are consistent with standard scaling $\zeta_{0}^{*}, \zeta_{0}^{\prime} \simeq 1$ as $t_{0} / t_{f} \rightarrow 0$. The standard scaling parameter $\beta$ depends only weakly on $t_{0} / t_{f}$. This effect is included in our uncertainty and is smaller than the statistical fluctuations. More information is given in the Supplemental Material [37].

We also explore the possibility of a small drift away from standard scaling in the residuals, by using the length density parameter estimator,

$$
\hat{\zeta}=\ell_{s}\left(t-t_{0}\right)^{2} / \mathcal{V}=\left(t-t_{0}\right)^{2} / \xi^{2}
$$

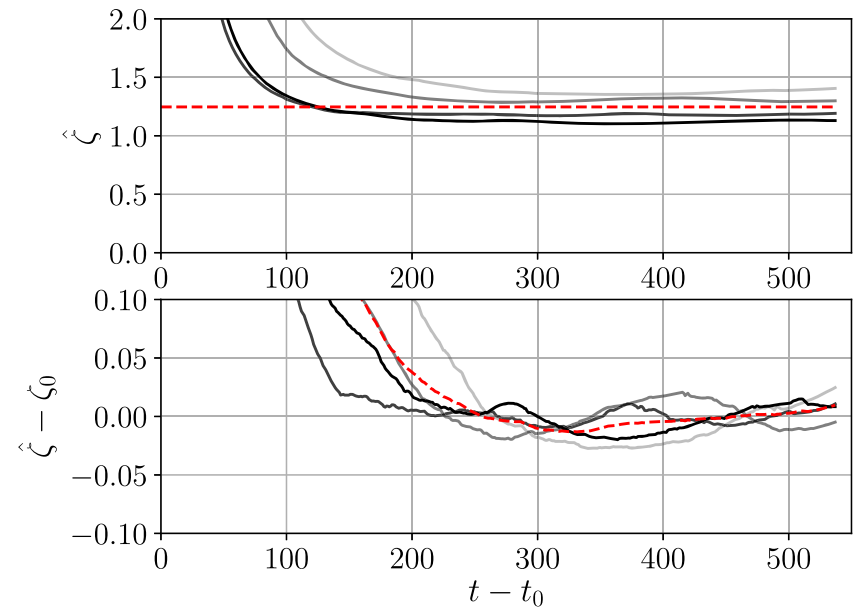

FIG. 2. Top: String length density parameter $\hat{\zeta}$ [see Eq. (5)] plotted against offset time $t-t_{0}$, for all $4 k$ runs with initial correlation length $\ell_{\phi}=5$. The dashed line shows the mean of $\zeta_{0}=1 / 4 \beta^{2}$. Bottom: Residuals $\left(\hat{\zeta}-\zeta_{0}\right)$ plotted against offset time $t-t_{0}$, for the same simulations. Individual runs are shown in solid lines and the residuals between the mean $\zeta_{0}$ and $\hat{\zeta}$ in dashed lines. where $t_{0}$ is the best fit value from the fit Eq. (5) for $\xi(t)$. In Fig. 2 we plot $\hat{\zeta}$ against $t-t_{0}$ for the 4 runs with $\ell_{\phi} \eta=5$. The figure gives a clear impression of $\hat{\zeta}$ tending to an asymptotically constant value. The residuals to the standard scaling fit for $\ell_{\phi} \eta=5$ are also shown in Fig. 2, with the mean shown as a dashed line. We fit the residual to a constant plus a logarithm according to

$$
\hat{\zeta}(t)-\zeta_{0}=\zeta_{r}+\alpha_{r} \log (\eta t),
$$

where $\alpha_{r}$ and $\zeta_{r}$ are fitting parameters, fitted over the four ranges in conformal time described earlier.

The measured values of $\zeta_{r}$ and $\alpha_{r}$ are given in Table III, along with the uncertainties. They are consistent with zero and give a tight bound on any logarithmic growth in the string length density parameter in the residual.

Having determined that standard scaling is the best model, we explore the uncertainty due to the finite lattice volume. We average the fit parameters over initial correlation lengths and fit ranges at each lattice size, and then perform a linear extrapolation in $1 / L \eta$. Our final result for the length density parameter is as follows [38]:

$$
\zeta_{0}=1.19 \pm 0.20 \text {. }
$$

The coefficient of any logarithm in the residuals is

$$
\alpha_{r}=(-0.04 \pm 0.30) \times 10^{-2},
$$

consistent with zero. The dominant error is statistical.

Conclusions.-In this Letter we have investigated the scaling density of axion strings, prompted by recent claims of a logarithmic increase in the string length density parameter $\zeta$ [24-30].

We have fitted the string length density from our simulations with three two-parameter models: the standard scaling model with the usual time offset $t_{0}$ to account for the initial string evolution and an asymptotically constant length density parameter $\zeta_{0}$; a model with a logarithmically increasing $\zeta$; and a model with an inverse-logarithmic correction. By linear fits to the mean string separation $\xi$, we obtain a well-determined result for the parameter $\zeta_{0}$, given in Eq. (11). The coefficients of the logarithm and inverse logarithm can be understood in terms of the dependence of $\zeta$ on the initial string evolution parameter 
$t_{0}$ and describe a disguised approach to scaling for nonzero $t_{0}$. We find they are consistent with zero when $t_{0} / t_{f} \rightarrow 0$, where $t_{f}$ is the final fitting time. The constant terms in the models are consistent with the standard scaling values. A search for a logarithmic correction to the residuals of the standard scaling model gives a tight upper bound on the magnitude of its coefficient Eq. (12): our $3 \sigma$ limit on a logarithmic correction to the string density parameter is $|\alpha|<0.94 \times 10^{-2}$.

We conclude that axion strings scale very well in the standard sense, and that between a $10^{12} \mathrm{GeV}$ PQ phase transition and the QCD transition at $100 \mathrm{MeV}$, any logarithmic correction to the string density parameter $\zeta_{0} \simeq 1$ must be less than about 0.5 .

An implication of the confirmation of standard scaling, important for network modeling [32], is that the energy loss rate per unit length of string must increase at the same rate as the effective string tension.

The tight constraint on the logarithmic correction also has implications for attempts to extend the dynamic range of global string simulations [28-30] by using frustrated strings [31]. Frustrated string models have fields with both global and local symmetries, and the string resembles a global string with an Abelian Higgs string at the core. The effect is to decouple the string tension $\mu$ and the axion decay constant $f_{a}$, so that $\kappa=\mu / \pi f_{a}^{2}$ can be chosen to be greater than 1 . As the effective tension of an axion string is $\mu_{a} \simeq \pi f_{a}^{2} \ln (\xi \eta)$, it was argued that a simulation with frustrated strings would effectively reach a string separation $\xi \sim \eta^{-1} \exp (\kappa)$.

It was found that there was an increase in the length density parameter $\zeta$ with the ratio $\kappa=\mu / \pi f_{a}^{2}$, apparently saturating at $\zeta \simeq 20$ around $\kappa \simeq 50$ [30]. This is far above our $O(1)$ upper bound on $\zeta$ at the QCD scale, casting doubt on the effectiveness of frustrated strings as a generic model of axion strings at large separations. Hence, one should not extrapolate the axion number density $n_{\text {ax }}$ to $\kappa \sim 70$. From Fig. 6 (right) of Ref. [30] one can estimate that $n_{\mathrm{ax}} / n_{\text {mis }} \simeq 0.5$ at $\kappa=1$, where $n_{\text {mis }}$ is the angle-averaged number density produced by the misalignment mechanism [5,30,39-41]. This is consistent with the directly measured values reported by other groups $[20,26]$. This value is about $60 \%$ of the extrapolated value [30], suggesting that the value of the axion dark matter mass of about $25 \mu \mathrm{eV}$ [30] should be revised upward by about 50\% in scenarios based on PQ symmetry breaking by a gauge singlet. We leave a more precise estimate for future work.

Finally, we note that frustrated string models [28-30] may be viable if the PQ symmetry breaking is accompanied by the breaking of a $U(1)$ gauge symmetry. The difference in the axion dark matter mass estimates between the models implies that the detection of an axion and an accurate measurement of its mass could distinguish between them.
We are grateful for fruitful discussions with $\mathrm{M}$. Kawasaki, J. Redondo, K. Saikawa, T. Sekiguchi, G. Villadoro, M. Yamaguchi, and J. Yokoyama. M. H. acknowledges support from the Science and Technology Facilities Council (Grant No. ST/L000504/1). J. L. and J. U. acknowledge support from Eusko Jaurlaritza (IT-97916) and MCIU/AEI/FEDER grant Fondo Europeo de Desarrollo Regional (Grant No. PGC2018-094626-BC21). A. L.-E. is supported by the Academy of Finland Grant No. 286769. A. L.-E. is grateful to the Early Universe Cosmology group of the University of the Basque Country for their generous hospitality and useful discussions. This work has been possible thanks to the computational resources on the STFC DiRAC HPC facility obtained under the dp116 project. Our simulations also made use of facilities at the i2Basque academic network and CSC Finland.

*mark.hindmarsh@helsinki.fi

joanes.lizarraga@ehu.eus

*asier.lopezeiguren@helsinki.fi

jon.urrestilla@ehu.eus

[1] R. D. Peccei and H. R. Quinn, Phys. Rev. Lett. 38, 1440 (1977); Phys. Rev. D 16, 1791 (1977).

[2] S. Weinberg, Phys. Rev. Lett. 40, 223 (1978); F. Wilczek, Phys. Rev. Lett. 40, 279 (1978).

[3] J. E. Kim, Phys. Rev. Lett. 43, 103 (1979); M. A. Shifman, A. I. Vainshtein, and V. I. Zakharov, Nucl. Phys. B166, 493 (1980).

[4] A. R. Zhitnitsky, Yad. Fiz. 31, 497 (1980) [Sov. J. Nucl. Phys. 31, 260 (1980)]; M. Dine, W. Fischler, and M. Srednicki, Phys. Lett. 104B, 199 (1981).

[5] J. Preskill, M. B. Wise, and F. Wilczek, Phys. Lett. 120B, 127 (1983); L. F. Abbott and P. Sikivie, Phys. Lett. 120B, 133 (1983); M. Dine and W. Fischler, Phys. Lett. 120B, 137 (1983).

[6] R. L. Davis, Phys. Lett. 180B, 225 (1986).

[7] M. Hindmarsh and T. Kibble, Rep. Prog. Phys. 58, 477 (1995).

[8] A. Vilenkin and E.P.S. Shellard, Cosmic Strings and Other Topological Defects (Cambridge University Press, Cambridge, England, 2000).

[9] P. Sikivie, Phys. Rev. Lett. 48, 1156 (1982).

[10] H. Georgi and M. B. Wise, Phys. Lett. 116B, 123 (1982).

[11] E. P. S. Shellard and R. A. Battye, Phys. Rep. 307, 227 (1998).

[12] P. Sikivie, Lect. Notes Phys. 741, 19 (2008).

[13] J.E. Kim and G. Carosi, Rev. Mod. Phys. 82, 557 (2010).

[14] M. Yamaguchi, M. Kawasaki, and J. Yokoyama, Phys. Rev. Lett. 82, 4578 (1999).

[15] M. Yamaguchi, M. Kawasaki, and J. Yokoyama, in Proceedings of the 7th International Symposium on Particles, Strings and Cosmology (PASCOS 99), Lake Tahoe, 1999 (World Scientific, Singapore, 1999), pp. 291-294. 
[16] M. Yamaguchi, M. Kawasaki, and J. Yokoyama, in Proceedings of the 3rd International Workshop on the Identification of Dark Matter (IDM 2000), York, United Kingdom, 2000 (World Scientific, Singapore, 2000), pp. 297-304.

[17] M. Yamaguchi and J. Yokoyama, Phys. Rev. D 67, 103514 (2003).

[18] T. Hiramatsu, M. Kawasaki, T. Sekiguchi, M. Yamaguchi, and J. Yokoyama, Phys. Rev. D 83, 123531 (2011).

[19] T. Hiramatsu, M. Kawasaki, K. Saikawa, and T. Sekiguchi, Phys. Rev. D 85, 105020 (2012); 86, 089902(E) (2012).

[20] M. Kawasaki, K. Saikawa, and T. Sekiguchi, Phys. Rev. D 91, 065014 (2015).

[21] A. Lopez-Eiguren, J. Lizarraga, M. Hindmarsh, and J. Urrestilla, J. Cosmol. Astropart. Phys. 07 (2017) 026.

[22] C. J. A. P. Martins and E. P. S. Shellard, Phys. Rev. D 54, 2535 (1996).

[23] C. J. A. P. Martins and E. P. S. Shellard, Phys. Rev. D 65, 043514 (2002).

[24] M. Gorghetto, E. Hardy, and G. Villadoro, J. High Energy Phys. 07 (2018) 151.

[25] M. Kawasaki, T. Sekiguchi, M. Yamaguchi, and J. Yokoyama, Prog. Theor. Exp. Phys. (2018), 091E01.

[26] A. Vaquero, J. Redondo, and J. Stadler, J. Cosmol. Astropart. Phys. 04 (2019) 012.

[27] M. Buschmann, J. W. Foster, and B. R. Safdi, arXiv:1906 .00967.

[28] L. Fleury and G. D. Moore, J. Cosmol. Astropart. Phys. 01 (2016) 004.
[29] V. B. Klaer and G. D. Moore, J. Cosmol. Astropart. Phys. 10 (2017) 043.

[30] V. B. Klaer and G. D. Moore, J. Cosmol. Astropart. Phys. 11 (2017) 049.

[31] C. T. Hill, A. L. Kagan, and L. M. Widrow, Phys. Rev. D 38, 1100 (1988).

[32] C. J. A. P. Martins, Phys. Lett. B 788, 147 (2019).

[33] D. Daverio, M. Hindmarsh, and N. Bevis, arXiv:1508 .05610 .

[34] W. H. Press, B. S. Ryden, and D. N. Spergel, Astrophys. J. 347, 590 (1989).

[35] T. Vachaspati and A. Vilenkin, Phys. Rev. D 30, 2036 (1984).

[36] N. Bevis, M. Hindmarsh, M. Kunz, and J. Urrestilla, Phys. Rev. D 82, 065004 (2010).

[37] See Supplemental Material at http://link.aps.org/ supplemental/10.1103/PhysRevLett.124.021301 for the results of the simulations and the fits to various fitting parameters for all simulations sizes $(1 k, 2 k$, and $4 k)$, for several values of $1 / \phi$ and $t_{0} / t_{f}$. We also show this results for simulations with constant comoving width.

[38] We have also performed simulation with constant comoving width, and observe a similar behavior, with $\zeta_{0}=$ $1.34 \pm 0.22$; see the Supplemental Material [37].

[39] K. J. Bae, J.-H. Huh, and J. E. Kim, J. Cosmol. Astropart. Phys. 09 (2008) 005.

[40] O. Wantz and E. P. S. Shellard, Phys. Rev. D 82, 123508 (2010).

[41] S. Borsanyi et al., Nature (London) 539, 69 (2016). 